\title{
Geomorphological and spectrophotometric analysis of Seth's circular niches on comet 67P/Churyumov-Gerasimenko using OSIRIS images
}
A. Lucchetti, ${ }^{1 \star}$ M. Pajola, ${ }^{2}$ S. Fornasier, ${ }^{3}$ S. Mottola,${ }^{4}$ L. Penasa, ${ }^{5}$ L. Jorda, ${ }^{6}$
G. Cremonese, ${ }^{1}$ C. Feller, ${ }^{3}$ P. H. Hasselmann, ${ }^{3}$ M. Massironi, ${ }^{7}$ S. Ferrari, ${ }^{5}$ G. Naletto, ${ }^{5,8,9}$ N. Oklay, ${ }^{4}$ H. Sierks, ${ }^{10}$ C. Barbieri, ${ }^{2,11}$ P. L. Lamy, ${ }^{6}$ R. Rodrigo, ${ }^{12,13}$ D. Koschny, ${ }^{14}$ H. Rickman, ${ }^{15,16}$ H. U. Keller, ${ }^{17}$ J. Agarwal, ${ }^{10}$ M. F. A'Hearn, ${ }^{18}$ M. A. Barucci, ${ }^{3}$ J. L. Bertaux, ${ }^{19}$ I. Bertini, ${ }^{11}$ S. Boudreault, ${ }^{10}$ V. Da Deppo, ${ }^{9}$ B. Davidsson, ${ }^{20}$ S. Debei, ${ }^{21}$ M. De Cecco, ${ }^{22}$ J. Deller, ${ }^{10}$ M. Fulle, ${ }^{23}$ O. Groussin, ${ }^{6}$ P. J. Gutierrez, ${ }^{24}$ C. Güttler, ${ }^{10}$ M. Hoffman, ${ }^{10}$ S. F. Hviid, ${ }^{4}$ W. H. Ip, ${ }^{25}$ J. Knollenberg, ${ }^{4}$ J. R. Kramm, ${ }^{10}$ E. Kührt, ${ }^{4}$ M. Küppers,${ }^{26}$ L. M. Lara, ${ }^{24}$ M. Lazzarin,,${ }^{11}$ F. La Forgia, ${ }^{11}$ L. Z. Lin, ${ }^{24}$ J. J. Lopez Moreno, ${ }^{24}$ F. Marzari, ${ }^{11}$ F. Preusker, ${ }^{4}$ F. Scholten, ${ }^{4}$ X. Shi, ${ }^{10}$ N. Thomas, ${ }^{27}$ C. Tubiana ${ }^{10}$ and J. B. Vincent ${ }^{4}$
Affiliations are listed at the end of the paper

Accepted 2017 June 21. Received 2017 June 8; in original form 2017 March 29

\begin{abstract}
We provide a detailed geomorphological and spectrophotometric analysis of the circular niches located on the Seth region of 67P using OSIRIS images. The features can be related to landslide events that occurred on 67P and shaped its surface, as the recent Aswan cliff collapse detected in the same region. We therefore provide an analysis of the area pre- and post-perihelion suggesting that no specific changes have been observed. To assess this, after performing a geomorphological map of the area that allows us to identify different terrain units, we computed the boulders cumulative size frequency distribution (SFD) of the niches, before and after the perihelion passage. The niches SFDs are characterized by a similar trend with two different power-law indices within the same deposit: lower power-law value (between -2.3 and -2.7 ) for boulders smaller than $5 \mathrm{~m}$ and steeper power-law value (between -4.7 and -5.0 ) for boulders larger than $5 \mathrm{~m}$. These trends suggest that smaller boulders have evolved and progressively have been depleted (lower power-law index), while bigger boulders are more representative of the event that generated the deposit and are less degraded. Then, we perform the spectrophotometric analysis of this region comparing pre- and post-perihelion results. We found colour changes within the area, in particular brighter patches related to the presence of exposed water ice mixed to the refractory materials have been detected in the post-perihelion images.
\end{abstract}

Key words: methods: data analysis - comets: general - comets: individual: 67P/ChuryumovGerasimenko.

\section{INTRODUCTION}

ESA's Rosetta spacecraft orbited closely around comet 67P/Churyumov-Gerasimenko (hereafter 67P) during its $2 \mathrm{yr}$ mission. From 2014 August to 2016 September, the camera system

${ }^{\star}$ E-mail: alice.lucchetti@oapd.inaf.it
Optical, Spectroscopic, and Infrared Remote Imaging System (OSIRIS; Keller et al. 2007) onboard Rosetta observed the comet nucleus down to few centimetres per pixel providing detailed images of the comet's surface and activity. The study of 67P morphology has been performed with unprecedented detail thanks to the OSIRIS image spatial coverage and resolution. A first OSIRIS analysis on the nucleus structure, morphology and jets activity is reported in Sierks et al. (2015) and Thomas et al. (2015). The comet's 

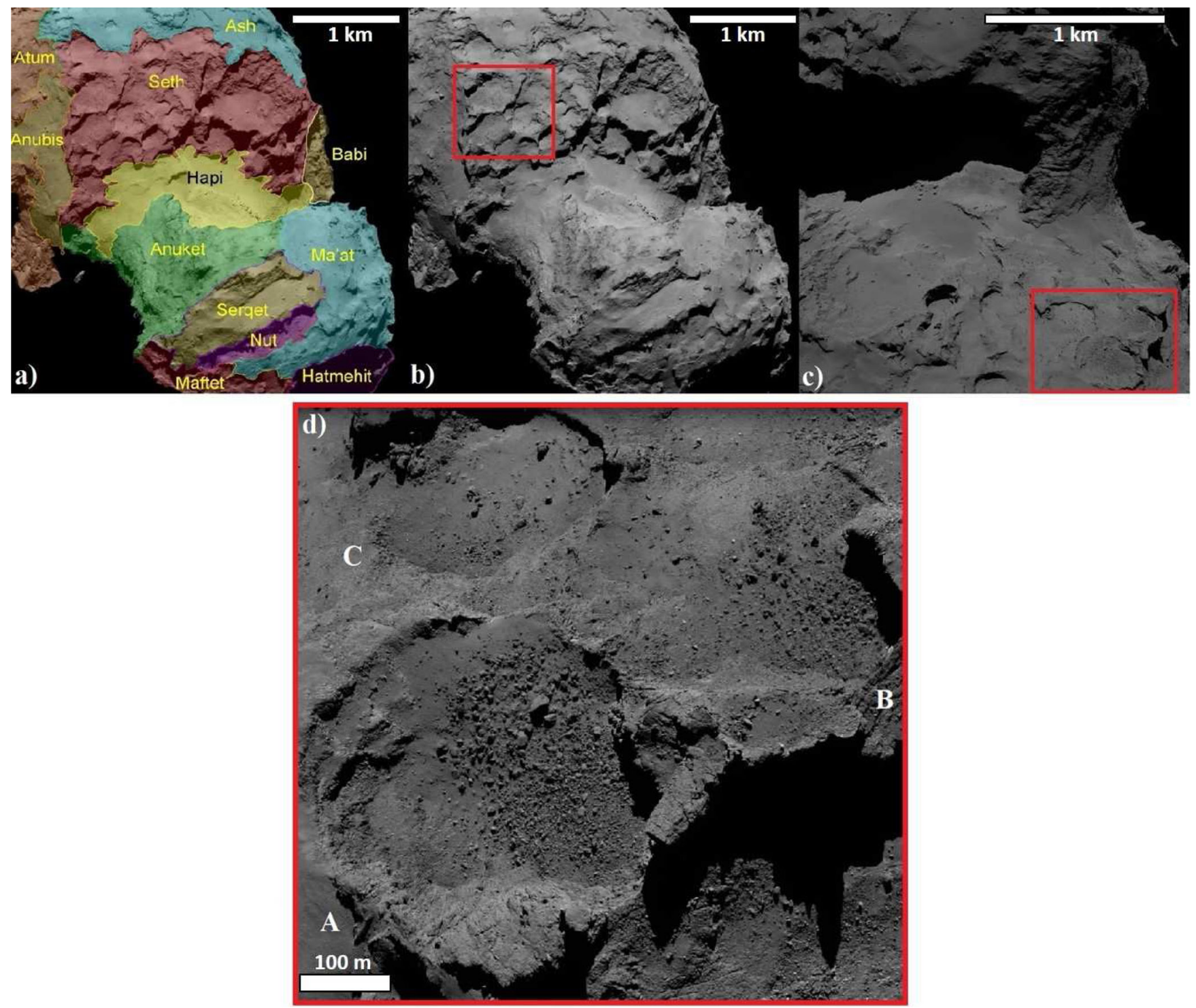

Figure 1. Geological context of the Seth region and the circular niches analysed in this work. (a) NAC image acquired in 2014 August 6 showing regional boundaries and nomenclatures of the comet 67P. The scale of the image is $2.2 \mathrm{~m} \mathrm{px}^{-1}$ and the Seth region is coloured in red. (b) The same NAC image displaying the circular niches analysed that are marked with the red square. (c) NAC image taken in 2014 August 21 (scale of $1.2 \mathrm{~m} \mathrm{px}^{-1}$ ) showing a different view of the circular niches, which are indicated by the red square. (d) The high-resolution WAC image acquired in 2016 August 24 (scale of $0.31 \mathrm{~m} \mathrm{px}^{-1}$ ) exhibiting the circular niches under study. We refer to the three niches as niches A, B and C as outlined in the image.

surface revealed a wide diversity in morphology such as layering (Massironi et al. 2015), pits (Vincent et al. 2015), boulders and fractures (Pajola et al. 2015, 2016c; El-Maarry et al. 2015a), and high reflectivity boulders (Pommerol et al. 2015). During the entire mission, surface changes have been detected thanks to images acquired before and after perihelion passage (Groussin et al. 2015; Pajola 2017; El-Maarry 2017). The global spectrophotometric properties of the comet were investigated in detail by Fornasier et al. (2015) identifying three different groups of terrains depending on spectral slope values. In addition to a global characterization of the comet, OSIRIS allowed a detailed analysis of specific regions identified on the comet by means of high resolution and multifilter images (La Forgia et al. 2015; Deshapriya et al. 2016; Lucchetti et al. 2016; Oklay et al. 2016a; Pajola et al. 2016d; Oklay et al. 2017). We decided to follow the approach of characterizing at the highest resolution possible specific regions of interest (ROIs) on 67P. We therefore performed a detailed analysis on an area belonging to the Seth region (El-Maarry et al. 2015b) that is characterized by flat-floored and steep-walled circular depressions (El-Maarry et al. 2015b; Giacomini et al. 2016). We focused our attention on the circular niches of the Seth area (Fig. 1) and performed a multidisciplinary study of these structures investigating their geomorphological and spectrophotometric properties. In addition, thanks to images acquired pre- and post-perihelion, we conducted a comparative analysis to find if this area has been subjected to surface changes. This can be useful to provide constraints about Seth's niches properties as well as their possible origin being one of the interesting features located on the surface of 67P. Specifically, the circular niches deposits can be considered the result of landslide events that occurred on the comet surface, as the recent Aswan cliff collapse (Pajola 2017), where it has been reported the occurring of falling material from the adjacent cliff after the Rosetta perihelion passage. Hence, 
with this work we plan to understand if the formation of these Seth's circular niches occurred recently or if it is correlated to older events that have shaped the comet's surface.

The paper is organized as follows. We perform a geomorphological analysis of the Seth circular niches identifying the different types of terrain units, then we derive the size frequency distribution (SFD) of boulders $\geq 1.8 \mathrm{~m}$ located in the different considered niches. The overall analysis has been performed making use of the gravitational slope map and the erosion and insolation model of the area, which have been calculated on the high-resolution digital terrain model (DTM). In addition, we compare for one of the niches the boulders SFD derived in images acquired pre- and post-perihelion to observe if there have been any changes. Finally, we present a spectrophotometric analysis of the area before and after the Rosetta perihelion passage to describe the colour variation of the niches and to detect possible colour changes.

\section{DATA SET AND METHODS}

The OSIRIS instrument was equipped with a narrow-angle camera (NAC), designed for the mapping of the comet's surface with 12 filters covering the spectral range of $250-1000 \mathrm{~nm}$ in high spatial resolution, and a wide-angle camera (WAC), which was optimized for the gas and dust studies in the vicinity of the comet with a combination of 14 filters in the spectral range of $240-720 \mathrm{~nm}$ (Keller et al. 2007). Our analysis of Seth's niches was performed using several OSIRIS images, including both NAC and WAC images, with different spatial resolutions. The WAC image used to obtain a geomorphological map of the area (Fig. 1d) was taken in 2016 August 24, during one of the last elliptical orbits of Rosetta. This high-resolution image gives a unique view of the circular niches of the Seth region permitting the identification of different terrain units such as talus deposits, outcropping materials and gravitational accumulation deposits. It was taken at a distance of $3 \mathrm{~km}$, resulting in a scale of $31 \mathrm{~cm} \mathrm{px}^{-1}$ and allowing the identification of features larger than $1 \mathrm{~m}$ located in the niches A and B (Fig. 1d). In this image, there is also a smaller niche, niche $\mathrm{C}$, whose orientation is not optimal for boulder counting; however, we consider it into the geomorphological analysis and the spectrophotometric modelling in order to provide a complete description of the area. To determine if this area was affected by surface changes during the lifetime of Rosetta, we used a NAC image taken before perihelion on 2015 March 28, that allows us to count boulders in niche A and to compare the cumulative SFD with the one obtained after perihelion. We made use of ARCGIS software to define the different terrain units characterizing the three niches and to select the boulders, which are defined as positive relief detectable in various images obtained with different observation geometries, with the constant presence of an elongated shadow (if the phase angle is greater than $0^{\circ}$; Pajola et al. 2015). We selected boulders following the approach of Pajola et al. (2015, 2016 b,c), i.e. by manually identifying these features as polygonal shapes and deriving their maximum length, i.e. the diameter and the corresponding area. The lowest boulders size considered for our analysis is a value above the three pixel sampling, to minimize the likelihood of misidentifications (Nyquist 1928), even if we are able to identify also smaller features from their elongated shadow since the image was taken with a phase angle of $70^{\circ}$. Anyway, we did not include boulders smaller than $5 \mathrm{px}$ in the statistical analysis because the slope of the SFD naturally drops off approaching the three pixel detection limit (Mazrouei, Daly \& Barnouin 2014; Michikami et al. 2008). In addition, to obtain the cumulative SFD $\mathrm{km}^{-2}$, we

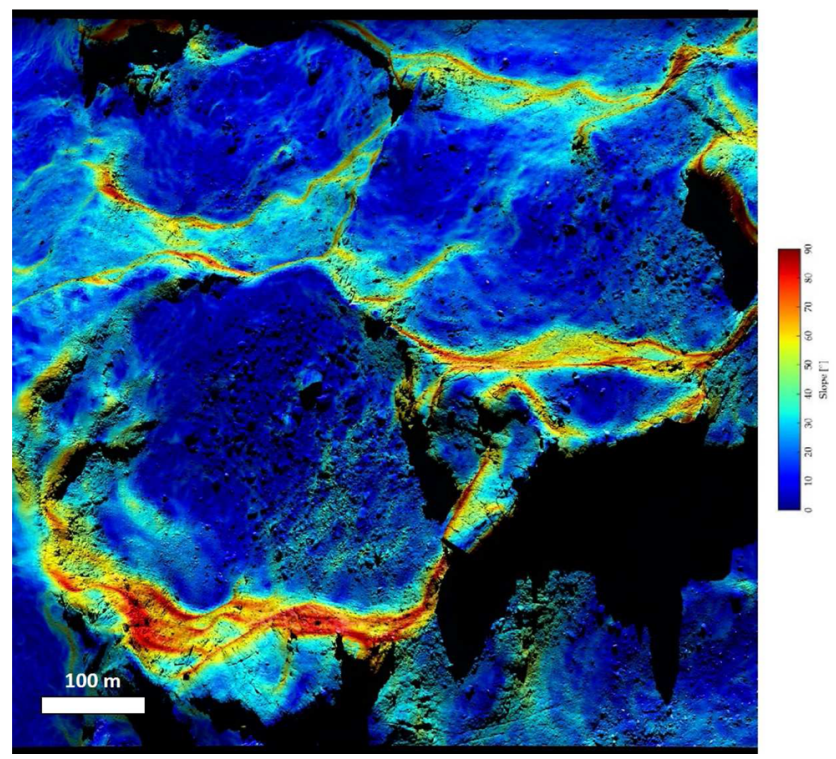

Figure 2. Gravitational slope map of Seth's niches obtained using the SPG (Preusker et al. 2015) shape model and the gravitational potential model derived assuming a homogeneous nucleus and accounting for the centrifugal force due to the nucleus rotation.

computed the area of each niche extracted from the 3D shape model of 67P (Preusker et al. 2015).

To describe in detail the area, we computed the gravitational slope from the 3D shape model derived with the stereo-photogrammetry (SPG) technique (Preusker et al. 2015) assuming a homogeneous nucleus with a density of $532 \mathrm{~kg} \mathrm{~m}^{-3}$ (Jorda et al. 2016), following the theory of Werner \& Scheeres (1997) and accounting for the centrifugal force due to the comet rotation (Rossi \& Fulchignoni 1999). The gravitational framework of the area (Fig. 2) was obtained in order to correlate the slope with the identified terrain unit and possibly identify the location from where boulders fell down. The gravitational slope is defined as the angle between the local surface normal pointing inside the nucleus and the local acceleration vector, which comprises both gravitation and centrifugal accelerations. The accumulation deposits areas show slopes $\leq 20^{\circ}$, while outcropping walls show slopes $\geq 50^{\circ}$.

To support our analysis, a specific high-resolution DTM was created on the basis of the entire NAC data set that provided multiple stereo coverage of the cometary surface. The DTM was derived with the stereo-photoclinometry (SPC) technique (Jorda et al. 2016) with a spatial resolution of $1 \mathrm{~m}$ (Fig. 3). Moreover, on the basis of the high-resolution DTM, an insolation and erosion model was calculated in order to have the total energy absorbed during a comet period and extrapolate the integrated erosion rate that characterizes niches A and B (Fig. 4). The insolation model used for our analysis is described in Keller et al. (2015) and is derived approximating the shape of 67P's circular niches through a polyhedron made of 300000 triangular facets, which is obtained by decimating a highresolution model based on images acquired during the orbital phase of Rosetta. The insolation of a facet is derived based on its orientation with respect to Sun. The erosion rate is referred to pure ice, however, the real erosion rate can be calculated considering the dust-to-ice ratio of the cometary material.

Finally, we consider a data set of images suitable to perform a detailed spectrophotometric analysis before and after the perihelion passage. The NAC images used are those acquired on 2014 November 11 at a distance of $44.8 \mathrm{~km}$ and on 2016 July 23 at a 


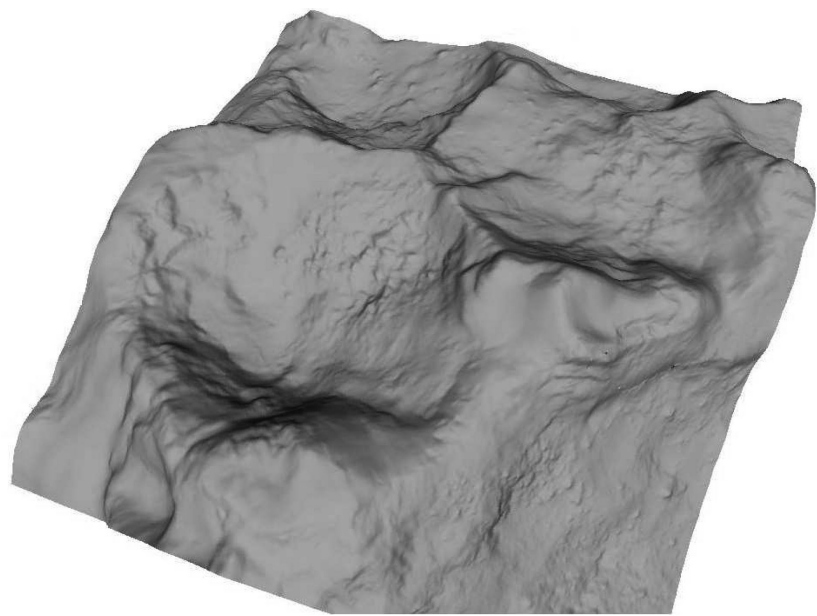

Figure 3. High-resolution DTM derived for the circular niches under study. The DTM was created with the SPC (Jorda et al. 2016) technique with a spatial resolution of $1 \mathrm{~m}$.
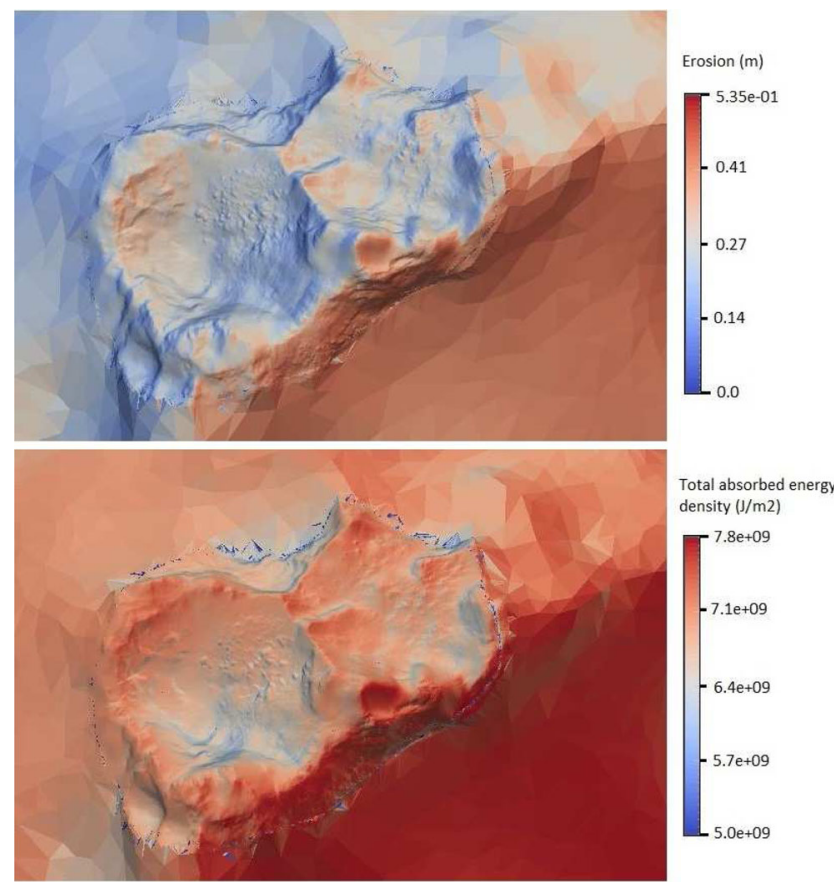

Figure 4. Upper panel: erosion integrated over one orbit, the maximum pure-ice erosion is $0.53 \mathrm{~m}$. Lower panel: insolation integrated over one orbit, the maximum of total absorbed energy is $7.8\left(\mathrm{~J} \mathrm{~m}^{-2}\right)$.

distance of $7.8 \mathrm{~km}$. The set of images used to derive spectrophotometric properties (that are in radiance units, $\mathrm{Wm}^{-2} \mathrm{~nm}^{-1} \mathrm{sr}^{-1}$, at the central wavelength of each filter) are converted into radiance factor (I/F) by using

$$
\frac{I}{F}=\frac{\pi I_{\mathrm{m}}}{F_{\odot}} r^{2}
$$

where $I_{\mathrm{m}}$ is the measured intensity in radiance units, $\frac{F \odot}{\pi}$ is the solar flux at 1 au measured at the central wavelength for each filter and $r$ is the heliocentric distance of the comet in astronomical units. The images were reduced using the OSIRIS standard pipeline up to level 3B, following the data reduction steps (Tubiana et al. 2015).
For the spectrophotometric analysis, the images of a given observing sequence were first coregistered taking the NAC orange filter (centred at $649.2 \mathrm{~nm}$ ) as reference and using a python script adapted from the code described in van der Walt et al. (2014) and the scikit-image library (van der Walt et al. 2014). For post-perihelion images acquired at higher spatial resolution, we also used the optical flow algorithm to improve the image coregistration (Farneback 2001). Then, images were photometrically corrected applying the Lommel-Seeliger disc law (Hapke 1993) and the geometric information about the illumination and observation angles were derived using the SPC shape model (Jorda et al. 2016). In the relative spectrophotometry, the flux of a given region of interest is integrated over a $3 \times 3$ pixels box and normalized at $535 \mathrm{~nm}$, following the same method described in Feller et al. (2016), Deshapriya et al. (2016), Fornasier et al. (2017) and Oklay et al. (2017).

The observation geometry, scale, phase angle and filters for each image used in the analysis are listed in Table 1.

\section{GEOMORPHOLOGICAL ANALYSIS}

\subsection{Geomorphological map}

The Seth region is located on the large lobe of the comet 67P and it is composed of a series of terraces alternated to steep walls showing outcrops of consolidated material (Giacomini et al. 2016). The origin of semicircular terraces is still unknown and can be related to collapse of material tending to form talus and rockfall-like deposits (Giacomini et al. 2016) or to the evolution of pits, the remnant of sinkhole collapse of buried cavities (Vincent et al. 2015). Niches and pit rims are not clearly discernible in some cases. Indeed, some of Seth's landforms can be the result of the interaction between the two origin processes: the original collapsed sinkhole occurred and the sublimation modified the landform shape with the consequent retreat of walls (Giacomini et al. 2016). This is indicated by the gravitational accumulation deposits detected at the foot of the walls (Pajola et al. 2015; Vincent et al. 2015). Between the different circular features present in this area, we chose three niches that exhibit large gravitational accumulation deposits, probably due only to the collapse of material from the outcrops walls. A geomorphological map of the area was performed highlighting the main geomorphological units, which are discriminated on the basis of the morphological properties following Giacomini et al. (2016) outlines (Fig. 5).

On the steep wall of the outcropping terrain, several fractures have been detected. Steep walls are found in correspondence with high gravitational slopes (see Fig. 2) larger than $40^{\circ}$ and usually show unconsolidated material at their foot. This is therefore interpreted as the result of collapses due to erosion by sublimation. We can provide a first hint regarding the different units characterizing the area because of the strong correlation with the slope obtained by gravity, indeed, the gravitational slope map can be fundamental to understand the limits of the different terrains presented in the geomorphological map. For instance, we found that areas with a slope below $20^{\circ}$ are representative of boulders deposits, while the gravitational slope of the outcropping walls are steeper than $40^{\circ}$, not allowing the boulders deposit to rest. In Fig. 5, the different deposits can be identified as taluses and gravitational accumulations, separated on the basis of their texture. They have the same gravitational source and are interpreted as falling material caused by erosion of the adjacent cliffs. Talus deposits are generally constituted by finer material often showing some larger blocks located all along the foot of the walls (Giacomini et al. 2016; Pajola et al. 
Table 1. List of OSIRIS images used in this work. The last column reports the specific analysis performed on the corresponding images.

\begin{tabular}{|c|c|c|c|c|c|c|c|}
\hline Date & UT & Distance $(\mathrm{km})$ & Scale $\left(\mathrm{m} \mathrm{px}^{-1}\right)$ & Phase angle $\left(^{\circ}\right)$ & Filter ID & $\lambda(\mathrm{nm})$ & Analysis \\
\hline NAC 2015-03-28 & 6.49 .49 & 29.3 & 0.55 & 68 & F82 Orange & 649.2 & Pre-perihelion boulders counting \\
\hline NAC 2014-11-14 & 22.07 .23 & 44.8 & 0.84 & 90 & F22 Orange & 649.2 & Pre-perihelion \\
\hline NAC 2014-11-14 & 22.07 .31 & 44.8 & 0.84 & 90 & F23 Green & 535.5 & spectrophotometric analysis \\
\hline NAC 2014-11-14 & 22.07 .55 & 44.8 & 0.84 & 90 & F28 Red & 743.7 & \\
\hline NAC 2014-11-14 & 22.08 .03 & 44.8 & 0.84 & 90 & F51 Ortho & 805.3 & \\
\hline NAC 2014-11-14 & 22.08 .11 & 44.8 & 0.84 & 90 & $\mathrm{~F} 61 \mathrm{Fe}_{2} \mathrm{O}_{3}$ & 931.9 & \\
\hline NAC 2016-07-23 & 07.30 .48 & 7.8 & 0.15 & 89 & F41 Near-IR & 882.1 & Post-perihelion \\
\hline NAC 2016-07-23 & 07.31 .00 & 7.8 & 0.15 & 89 & F22 Orange & 649.2 & spectrophotometric analysis \\
\hline
\end{tabular}

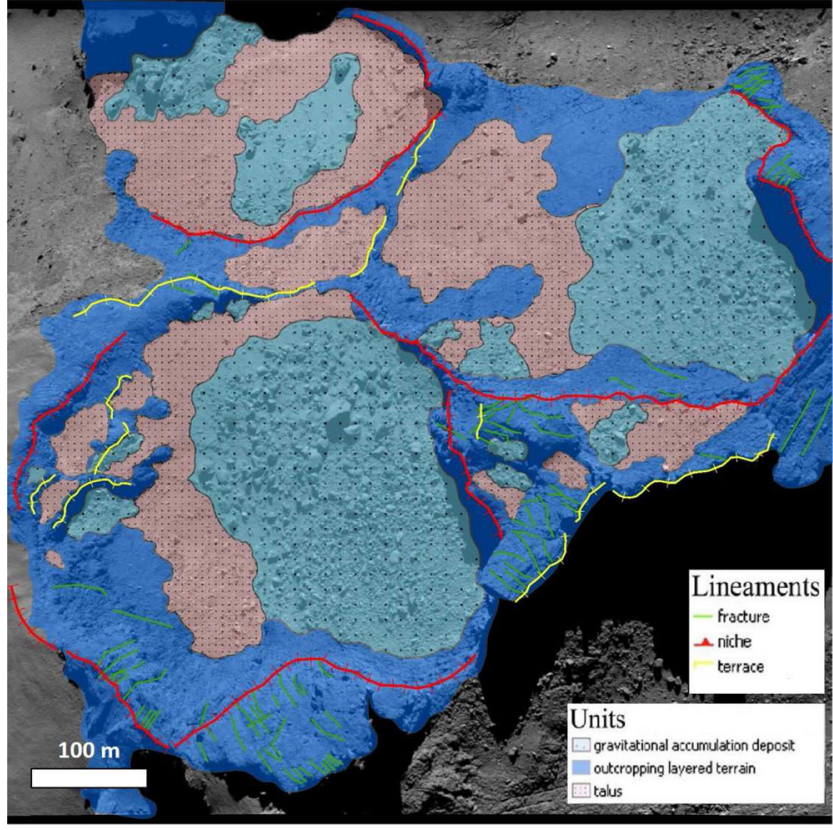

Figure 5. Geomorphological map of Seth's niches presenting the identified units and lineaments. This map was derived by using the WAC image taken on 2016 August 24 at a distance of $3 \mathrm{~km}$ from the comet. The corresponding scale is $0.31 \mathrm{~m} \mathrm{px}^{-1}$.

2016d) and are thought to be the result of the erosion of the material fallen from the adjacent cliffs. The gravitational accumulation deposits are composed by boulders of different dimensions and their source is well associated with the material detachment from the steep outcropping walls due to erosion by sublimation (La Forgia et al. 2015; Massironi et al. 2015; Oklay et al. 2016a; Oklay et al. 2017).

\subsection{Boulders analysis}

Using the WAC image acquired on 2016 August 24, we counted boulders located in niches $\mathrm{A}$ and $\mathrm{B}$. We do not consider niche $\mathrm{C}$ in this type of analysis since the orientation of the feature is not optimal to perform boulders counting. The total number of boulders counted on niche A is 2526, 2216 of which have diameters larger than $1.8 \mathrm{~m}$, while on niche B we counted 2313 boulders, with 2049 boulders larger than $1.8 \mathrm{~m}$. We derived the cumulative SFD $\mathrm{km}^{-2}$ using the corresponding area $\left(0.08\right.$ and $0.06 \mathrm{~km}^{2}$ for niches $\mathrm{A}$ and $\mathrm{B}$, respectively) calculated from the $3 \mathrm{D}$ shape model of 67P (Preusker et al. 2015). We then obtained the cumulative SFD of boulders using a constant bin size of $0.31 \mathrm{~m}$, which is approximately the pixel resolution of the WAC image used for the analysis. We found different behaviour on the basis of the boulders dimension obtaining a similar trend for both niches. Specifically, for niche A, we found a power-law index of $-2.3+0.1 /-0.1$ for diameter ranging from 1.8 to $5 \mathrm{~m}$ (2047 boulders) and a power-law index of $-5.0+0.2 /-0.1$ (169 boulders) for boulders diameter larger than $5 \mathrm{~m}$. The fitting regression line for niche B gives a power-law index of $-2.7+0.1 /-0.1$ for boulders diameter ranging from 1.8 to $5 \mathrm{~m}$ (1955 boulders) and a power-law index of $-4.7+0.2 /-0.1$ (94 boulders) for boulders diameter larger than $5 \mathrm{~m}$. This behaviour is shown in Fig. 6.

We outline that the fitting regression line used to obtain the powerlaw index does not consider those points that are equally cumulatively repeated because they represent a poor statistic that has not been considered by the fit. This effect has been already observed for bigger boulder sizes as presented in Pajola et al. (2015) and Michikami et al. (2008). The lower limit of boulder dimension is assumed to be 5 px because the slope of the SFD naturally drops off approaching the three pixel detection limit (Mazrouei et al. 2014; Michikami et al. 2008). The cumulative number of boulders larger than $1.8 \mathrm{~m}$ is $27000 \mathrm{~km}^{-2}$ and $29000 \mathrm{~km}^{-2}$ for niches A and B, respectively.

We compare our results with what was found in previous works, in particular Pajola et al. (2015) defined three possible explanations for boulders formation on the basis of a first analysis of SFD obtained for the Northern hemisphere of 67P. In addition, there have been many works that tried to interpret the different boulders fields located in various regions or localized areas of the comet (La Forgia et al. 2015; Pommerol et al. 2015; Lucchetti et al. 2016; Pajola et al. 2016a,c,d; Oklay et al. 2016a,b; Oklay et al. 2017). Generally, the behaviour of power-law indices reflects the process acting on the area analysed: (i) SFD with a power-law index ranging between 

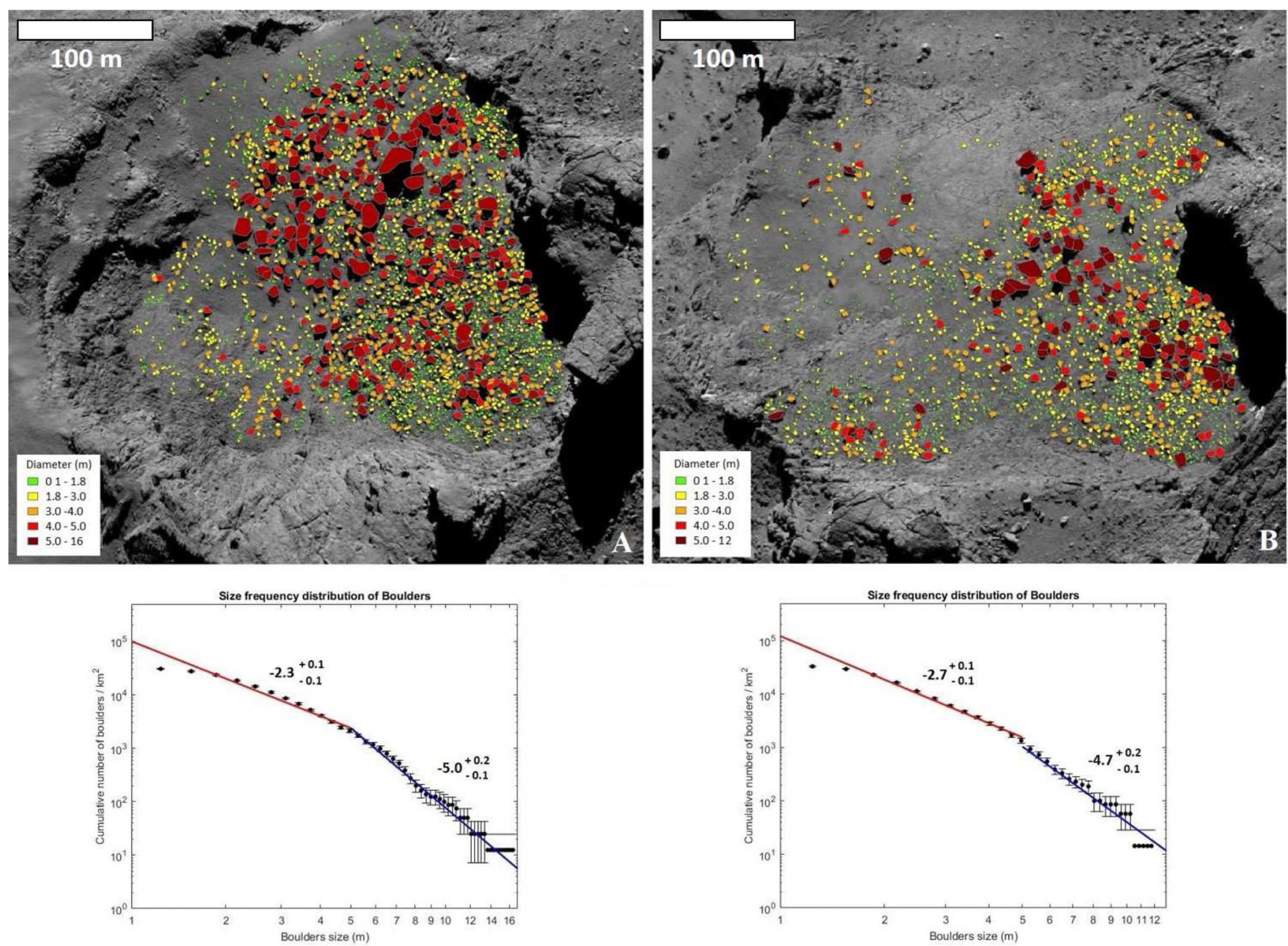

Figure 6. The upper panel shows the spatial distribution of boulders on niches A and B counted on the WAC image acquired on 2016 August 24 . Boulders smaller than $5 \mathrm{px}$, where the boulder size corresponds to the length calculated from the corresponding area, are in green and are not taken into account in the statistic. The lower panel shows the cumulative SFD of boulders larger than $5 \mathrm{px}$ identified on the two niches. The bin size is $0.31 \mathrm{~m}$ and vertical error bars indicate the root of the cumulative number of counting boulders (as from Michikami et al. 2008). The fitting regression line for niche A gives a power-law index of $-2.3+0.1 /-0.1$ for boulders diameter ranging from 1.8 to $5 \mathrm{~m}$ and a power-law index of $-5.0+0.2 /-0.1$ for boulders diameter larger than $5 \mathrm{~m}$. The fitting regression line for niche B gives a power-law index of $-2.7+0.1 /-0.1$ for boulders diameter ranging from 1.8 to $5 \mathrm{~m}$ and a power-law index of -4.7 $+0.2 /-0.1$ for boulders diameter larger than $5 \mathrm{~m}$.

-5.0 and -6.0 is indicative of high fragmentation resulting from a depression formation with subsequent escape of high-pressure volatile, (ii) SFD with power-law indices between -3.5 and -4.5 have been observed to be indicative of gravitational events triggered by sublimation and/or thermal fracturing causing regressive erosion and (iii) SFD with power-law indices ranging between -1.0 and -2.0 are representative of the evolution of the original material formed during both the collapsing event or the gravitational event, not particularly renewed, or present in areas where continuous and high sublimation occurred or is still occurring. In some specific cases, different SFD power-law indices have been identified than those described above. This is interpreted as a possible mixture of different processes occurring on such areas (Deshapriya et al. 2016).

For circular niches, we expect a similar behaviour to the SFD of case (ii) because of the geomorphology of the area, however, we found a different behaviour for both niches. For smaller boulders (diameter ranging between 1.8 and $5 \mathrm{~m}$ ) we found a power-law index of -2.3 and -2.7 for niches $\mathrm{A}$ and $\mathrm{B}$, respectively. This value is between the two power-law indices of cases (ii) and (iii) suggesting that the boulders formed during the gravitational event (usually characterized by a power-law index ranging from -3.5 to -4.5 ) have been evolved and progressively depleted lowering the power-law index of the SFD. In addition, the degradation of smaller boulders concurred to determine this behaviour. Hence, we can suggest that very degraded gravitational deposits show a lower power-law index maybe indicating that these deposits are older than the others. The power-law index describing the boulders larger than $5 \mathrm{~m}$ is instead characterized by a larger value, -5.0 and -4.9 for niches A and B, respectively, that can be related to the SFD case (i). The number of boulders considered in these SFDs is low, 169 and 94 boulders, for niches A and B, respectively, hence, the statistic can be affected by the small number of features counted. However, this different trend can be explained considering that bigger boulders are more representative of the event that raise the deposit and are less degraded than smaller boulders. Therefore, we expect higher power-law indices for bigger boulders and lower ones for smaller boulders within the same deposit, as found in our analysis.

We can also compare the results of niches $\mathrm{A}$ and $\mathrm{B}$ with what was found previously by Pajola et al. (2015) in the same area. The work reported the statistic analysis regarding boulders with a 

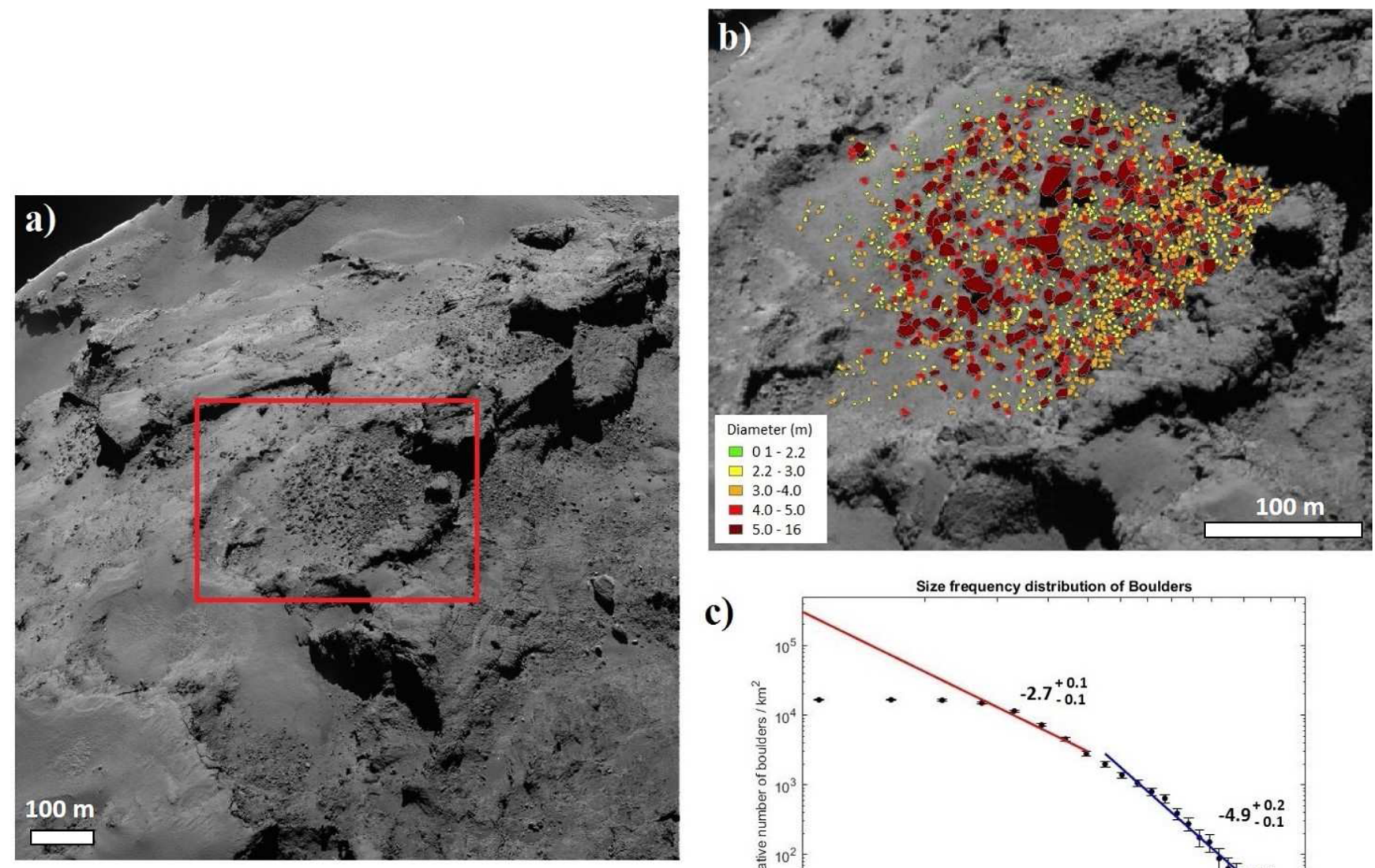

c)

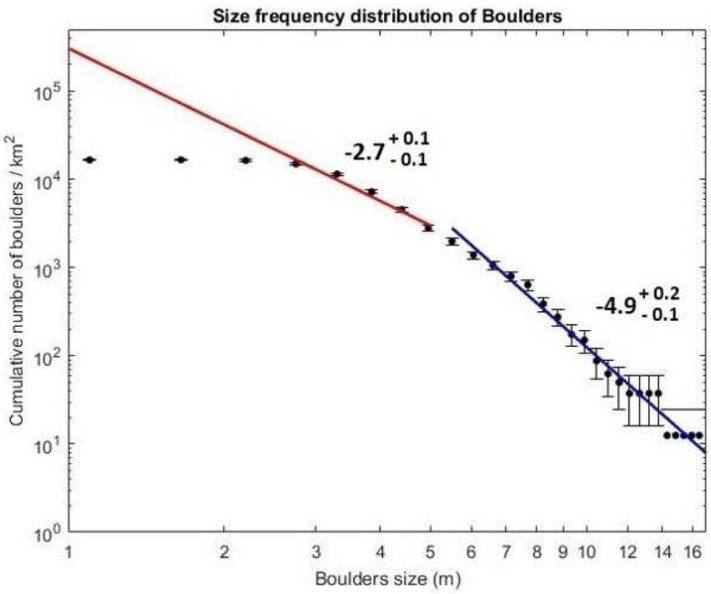

Figure 7. (a) The NAC image acquired on 2015 March 28 (resolution scale of $0.55 \mathrm{~m} \mathrm{px}^{-1}$ ) on which we perform the pre-perihelion analysis. The red box displays niche A on which we counted boulders. (b) The spatial distribution of boulders on niche A. Boulders smaller than 4 px, where the boulder size corresponds to the length calculated from the corresponding area, are in green and are not taken into account in the statistic. (c) The cumulative SFD of boulders larger than $4 \mathrm{px}$ identified on niche A. The bin size is $0.55 \mathrm{~m}$ and vertical error bars indicate the root of the cumulative number of counting boulders (as from Michikami et al. 2008). The fitting regression line gives a power-law index of $-2.7+0.1 /-0.1$ for boulders diameter ranging from 2.7 to $5 \mathrm{~m}$ and a power-law index of $-4.9+0.2 /-0.1$ for boulders diameter larger than $5 \mathrm{~m}$.

diameter larger than $7 \mathrm{~m}$ that are characterized by a power-law index of -6.5 and -5.5 for niches A and B, respectively. Pajola et al. (2015) explained the behaviour of these boulders field as the expression of high fragmentation resulting from a depression formation with subsequent escape of high pressure volatile [SFD case (iii)]. The existing difference with our work $(-5.0$ and -4.9 for boulders larger than $5 \mathrm{~m}$ for niches $\mathrm{A}$ and $\mathrm{B}$ ) can be explained by the fact that increasing the resolution of the image, the boulders counting improves allowing the identification of smaller boulders that enriched the statistic and slightly changed the SFD trend. To assess if there have been any changes in this area, we considered a NAC image taken before perihelion to have a direct comparison between the boulders identified. For this purpose, we took an image acquired on 2015 March 28 (see Table 1) that has a similar geometry of the WAC image, but contains only niche A. Therefore, we performed the boulder analysis identifying 1329 boulders (Fig. 7). This number is smaller than the previous one because of the different resolution. Considering a bin size of $0.55 \mathrm{~m}$ (equal to the image pixel resolution as in the previous case), the fitting regression line has a power-law index equal to $-2.7+0.1 /-0.1$ for boulders diameter ranging from 2.7 to $5 \mathrm{~m}$ ( 974 boulders) and a power-law index of $-4.9+0.2 /-0.1$ for boulders diameter larger than $5 \mathrm{~m}$ (225 boulders). Below $4 \mathrm{px}$ the distribution starts to roll over indicating that the boulder distribution is not complete. The number of boulders with a diameter larger than $2.7 \mathrm{~m}$ is 1199 and it is comparable with the number of boulders larger than $2.7 \mathrm{~m}$ in niche A of the WAC image, equal to 1148 . The SFD power-law indices obtained in images acquired pre- and post-perihelion are slightly in agreement, even if the SFD powerlaw index obtained with WAC image is little lower. This implies no particular changes in the area (in particular in niche A), at least from the geomorphological point of view and, in addition, it suggests that the source event of these gravitational deposits occurred in the past and is not related to the 67P recent detected activity, as found for Aswan cliff (Pajola 2017). This consideration is also supported by the results coming from the erosion and insolation model integrated over one 67P orbit (Fig. 4): the erosion rate characterizing the area has a maximum of only $0.53 \mathrm{~m}$ and does not strongly affect the area. However, it must be noted that the erosion mainly affects one 

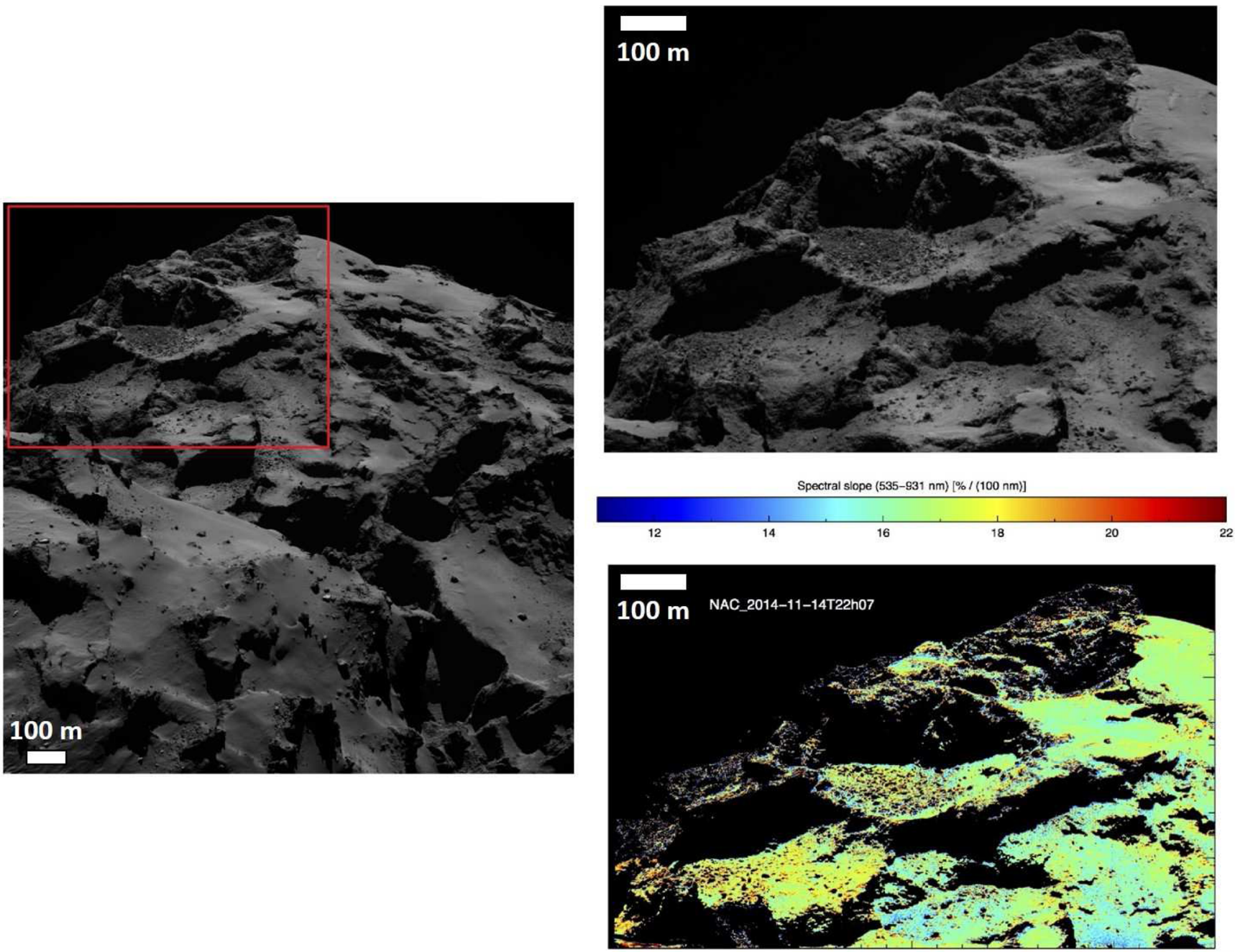

Figure 8. (a) The image acquired on 2014 November 11 used to perform the pre-perihelion analysis. The image was taken at a distance of $44.8 \mathrm{~km}$, resulting in a scale of $0.84 \mathrm{~m} \mathrm{px}^{-1}$. The area containing Seth's niches is outlined by the red box and its close out is shown in (b). (c) Spectral slope of the niches area computed in the $535-931 \mathrm{~nm}$ range, after the normalization at $535 \mathrm{~nm}$ and it is in per cent $(100 \mathrm{~nm})^{-1}$ unit. Sun is towards the top and the phase angle is $90^{\circ}$.

specific side of niches $\mathrm{A}$ and $\mathrm{B}$ from which we expect the material fell down in the past. In the last 67P orbit no gravitational collapses have been observed, but the constant presence of this selective erosion can result in future collapses coming from that specific side of the niches.

\section{SPECTROPHOTOMETRIC ANALYSIS}

We performed a spectrophotometric analysis on NAC images acquired before and after perihelion in order to characterize the colour changes after the Rosetta perihelion passage. For the pre-perihelion study, we chose a colour sequence centred on the Seth niches that was acquired on 2014 November 14 at phase angle of $90^{\circ}$, at a heliocentric distance of 2.97 au and with seven NAC filters (F22, F23, F24, F27, F28, F51 and F61). The distance between Rosetta and the comet at that time was $44.8 \mathrm{~km}$, giving a spatial resolution of $0.84 \mathrm{~m} \mathrm{px}^{-1}$ with the NAC camera. We evaluated the spectral slope in the 535-931 nm range normalizing at $535 \mathrm{~nm}$ (Fig. 8). The colour bar was chosen so that blue colour corresponds to regions with a lower slope than average, while the red colour represents regions with a higher slope than average. The average value obtained from a Gaussian fitting of the slope distribution is $16.6 \pm 1.4$ [ per cent $\left.(100 \mathrm{~nm})^{-1}\right]$. This value can be compared with the spectral slope analysis presented in Fornasier et al. $(2015,2016)$, even if it must be noted that in these studies the spectral slope was evaluated in the $535-882 \mathrm{~nm}$ range, but in the data set of 2014 November the $882 \mathrm{~nm}$ filter observation was missing. However, considering that the spectrum is usually linear in the $882-931 \mathrm{~nm}$ range and that the slope is evaluated normalizing at the same wavelength $(535 \mathrm{~nm})$, the variations related to the slightly different wavelength range used should be negligible. Fornasier et al. (2015) analysed the spectral slope from colour images acquired during 2014 July-August for the phase angle lower than $55^{\circ}$. They reported an important phase reddening for the nucleus of comet 67P, as well as colour heterogeneities at macro scale with the identification of three kinds of terrains: (i) terrains with the lower spectral slope [11-14 per cent $(100 \mathrm{~nm})^{-1}$ at phase angle $54^{\circ}$ ) mostly associated with regions showing activity and having a surface composition enriched in water ice, (ii) average slope terrains [having a spectral slope of 14-18 per cent $(100 \mathrm{~nm})^{-1}$ at phase angle $54^{\circ}$ ] and (iii) redder terrains [slope $>18$ per cent $(100 \mathrm{~nm})^{-1}$ at phase angle $54^{\circ}$ ] associated mostly to regions covered by dust. The Seth region was identified as belonging to the 

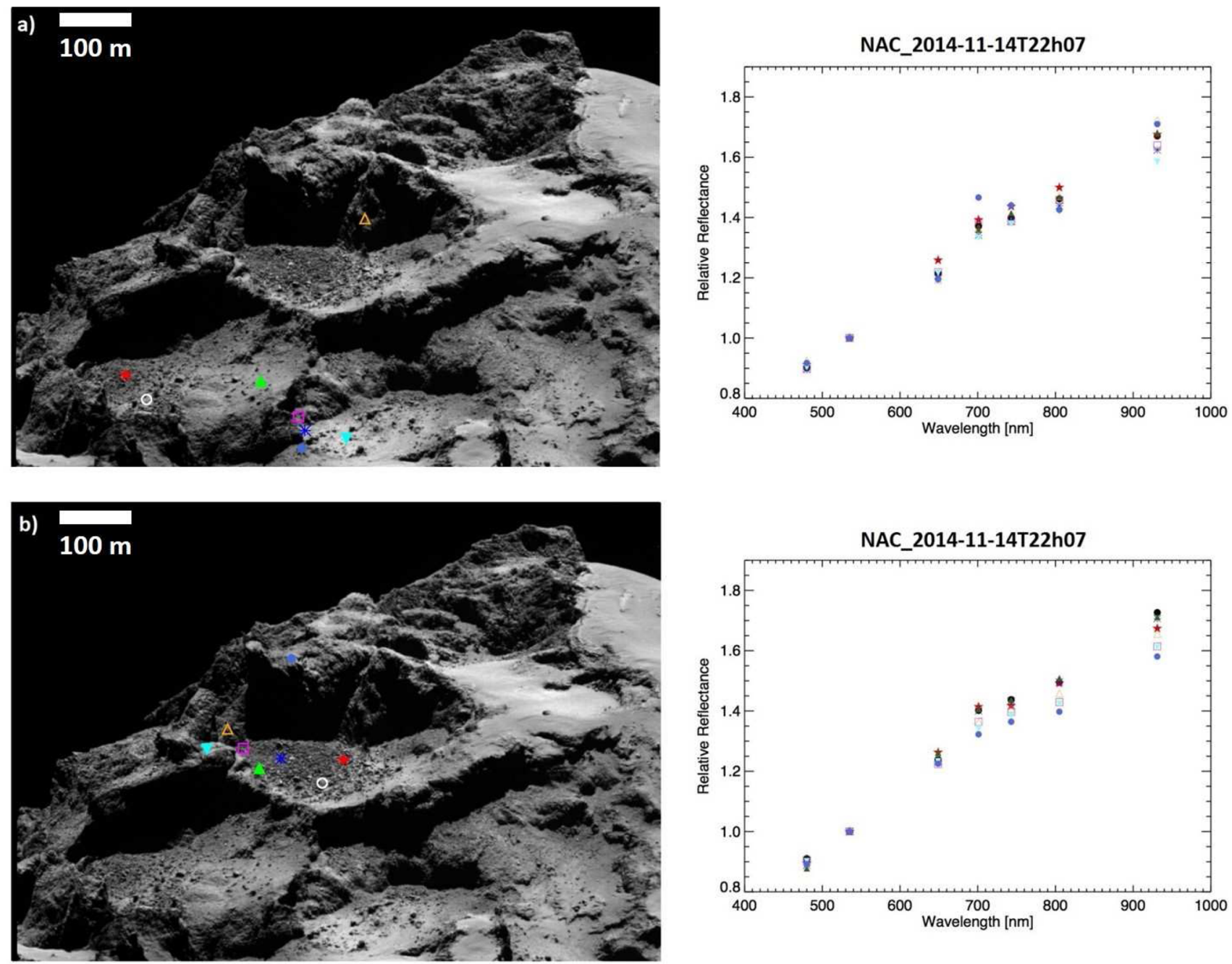

Figure 9. On the left are shown some selected ROIs of the pre-perihelion where the spectrophotometry has been computed. On the right, the relative reflectance for the corresponding selected regions is displayed, after normalization at $535 \mathrm{~nm}$. The black filled circle on the right is represented by a white not filled circle on the image on the left.

lower spectral slope group in Fornasier et al. (2015) analysis. More detailed analysis of Seth in high spatial resolutions are reported in Oklay et al. (2016b), where it is shown that this region is mainly active even if a large area is possibly mixed with the airfall material described in Thomas et al. (2015). The fact that Seth is relatively bluer is confirmed also by comparing the spectral slope here derived at $\alpha=90^{\circ}$ [16.6 per cent $(100 \mathrm{~nm})^{-1}$ ] with the one extrapolated at the same phase angle from the spectral reddening coefficients derived from 2014 July-August observations of the comet in the phase angle range $0^{\circ}-55^{\circ}$ [>19 per cent $(100 \mathrm{~nm})^{-1}$, see fig. S3 in Fornasier et al. 2016.

The comparison of the spectral slope map with the geomorphological units reveals a strong correlation with the morphological units identified, similarly to what was found in La Forgia et al. (2015). Indeed, Fig. 8 shows some local heterogeneities in the spectral slope value, with rough terrains having gravitational accumulation deposits which are spectrally redder, while talus deposits at the feet of the terraces are spectrally bluer. We thus investigate 16 different ROIs, as shown in Fig. 9. The ROIs represented by the blue asterisk/cyan triangle symbols in Fig. 9 a and by the blue circle and cyan triangle in Fig. 9 b are clear examples of relatively bluer terrains, associated with taluses deposits.
The spectrophotometry indicates a linear increasing of the reflectance with the wavelength, without any evidence of clear absorption bands. However, an enhancement of the reflectance at 700 and $743 \mathrm{~nm}$ is evident, especially in the shadowed area (as for the ROI represented by the blue circle in Fig. 9a, or red star in Fig. 9b). A similar spectral behaviour was observed by Fornasier et al. (2015) and attributed to cometary emissions (faint but visible especially on shadows on the nucleus) from the coma between the spacecraft and the 67P's nucleus. This implies that even at large heliocentric distance the comet was already active. The spectral slope values in the 535-931 nm range for the different ROIs investigated are shown in Table 2 .

The Seth region was also observed after the perihelion passage at a similar phase angle $\left(89^{\circ}\right)$ and a similar large outbound heliocentric distance (3.44 au) on 2016 July 23. At this time the spacecraft was much closer to the comet, at an altitude of $7.8 \mathrm{~km}$ giving a spatial resolution of about $0.15 \mathrm{~m}$. During these close observations, a couple of colour sequences were acquired at UT time 7h31 and $7 \mathrm{~h} 35$ using three NAC filters centred at 480, 649 and $882 \mathrm{~nm}$. Fig. 10 shows the RGB maps in false colours of the Seth region and the associated spectral slope maps. As most of the previous results on spectrophotometry of the $67 \mathrm{P}$ comet are normalized at 
Table 2. Spectral slope values for the ROIs investigated in Figs 9 (535$931 \mathrm{~nm}$ range), 11 and 12 (535-882 $\mathrm{nm}$ range).

\begin{tabular}{|c|c|c|}
\hline Figure & ROIs & Spectral slope $\left[\right.$ per cent $\left.(100 \mathrm{~nm})^{-1}\right]$ \\
\hline $9 \mathrm{a}$ & White circle & $16.9 \pm 0.3$ \\
\hline $9 \mathrm{a}$ & Red star & $17.1 \pm 0.3$ \\
\hline $9 \mathrm{a}$ & Blue asterisk & $15.8 \pm 0.3$ \\
\hline $9 \mathrm{a}$ & Green triangle & $17.2 \pm 0.3$ \\
\hline $9 \mathrm{a}$ & Magenta square & $16.1 \pm 0.3$ \\
\hline $9 \mathrm{a}$ & Orange triangle & $18.3 \pm 0.3$ \\
\hline $9 \mathrm{a}$ & Cyan upsidedown triangle & $14.8 \pm 0.3$ \\
\hline $9 \mathrm{a}$ & Royal blue circle & $17.9 \pm 0.3$ \\
\hline $9 \mathrm{~b}$ & White circle & $18.4 \pm 0.3$ \\
\hline $9 \mathrm{~b}$ & Red star & $17.0 \pm 0.3$ \\
\hline $9 \mathrm{~b}$ & Blue asterisk & $17.8 \pm 0.3$ \\
\hline $9 \mathrm{~b}$ & Green triangle & $18.0 \pm 0.3$ \\
\hline $9 \mathrm{~b}$ & Magenta square & $15.5 \pm 0.3$ \\
\hline $9 \mathrm{~b}$ & Orange triangle & $16.6 \pm 0.3$ \\
\hline $9 \mathrm{~b}$ & Cyan upsidedown triangle & $15.4 \pm 0.3$ \\
\hline $9 \mathrm{~b}$ & Royal blue circle & $14.7 \pm 0.3$ \\
\hline $11 \mathrm{a}$ & White circle & $16.2 \pm 0.4$ \\
\hline $11 \mathrm{a}$ & Red star & $-0.8 \pm 0.4$ \\
\hline $11 \mathrm{a}$ & Blue asterisk & $18.0 \pm 0.4$ \\
\hline $11 \mathrm{a}$ & Green triangle & $16.9 \pm 0.4$ \\
\hline $11 \mathrm{a}$ & Magenta square & $10.9 \pm 0.4$ \\
\hline $11 \mathrm{~b}$ & White circle & $14.8 \pm 0.4$ \\
\hline $11 \mathrm{~b}$ & Red star & $7.6 \pm 0.4$ \\
\hline $11 \mathrm{~b}$ & Blue asterisk & $9.2 \pm 0.7$ \\
\hline $11 \mathrm{~b}$ & Green triangle & $14.9 \pm 0.4$ \\
\hline $11 \mathrm{~b}$ & Magenta square & $0.9 \pm 0.7$ \\
\hline $12 \mathrm{a}$ & White circle & $15.5 \pm 0.4$ \\
\hline $12 \mathrm{a}$ & Red star & $13.1 \pm 0.4$ \\
\hline $12 \mathrm{a}$ & Blue asterisk & $3.2 \pm 0.4$ \\
\hline $12 \mathrm{a}$ & Green triangle & $14.6 \pm 0.4$ \\
\hline $12 \mathrm{a}$ & Magenta square & $15.7 \pm 0.4$ \\
\hline $12 \mathrm{~b}$ & White circle & $16.1 \pm 0.4$ \\
\hline $12 \mathrm{~b}$ & Red star & $15.5 \pm 0.4$ \\
\hline $12 \mathrm{~b}$ & Blue asterisk & $17.3 \pm 0.7$ \\
\hline $12 \mathrm{~b}$ & Green triangle & $9.1 \pm 0.4$ \\
\hline $12 \mathrm{~b}$ & Magenta square & $6.0 \pm 0.7$ \\
\hline
\end{tabular}

$535 \mathrm{~nm}$, we evaluate the spectral slope in the $882-535 \mathrm{~nm}$ range normalizing our data at $535 \mathrm{~nm}$ using a linear interpolation between the 480 and $649 \mathrm{~nm}$ observations. Compared to the pre-perihelion images, several bright and bluer regions close to shadows or in the shadows are evident, indicating a local enrichment in ice mixed to the refractory material.

We thus investigate the spectrophotometry of some ROIs (Figs 11 and 12) normalizing our data at $535 \mathrm{~nm}$, as done for the spectral slope maps. Several points clearly show a flat spectrophotometric behaviour or a spectrophotometry with spectral slope values much lower than the surrounding regions. In particular, the ROI indicated by the red star symbol in Fig. 11a, by the magenta square in Fig. $11 \mathrm{~b}$ and by the blue asterisk in Fig. $12 \mathrm{a}$ are spectrally bluer and they are consistent with the presence of exposed water ice mixed to the refractory materials, as previously observed in different regions of the nucleus (Fornasier et al. 2015; Pommerol et al. 2015; Barucci et al. 2016; Deshapriya et al. 2016; Filacchione et al. 2016; Fornasier et al. 2016; Oklay et al. 2016a,b; Pajola 2017; Oklay et al. 2017). The spectral slope values in the 535-882 nm range for the different ROIs investigated are shown in Table 2.
Despite the higher abundance of bluer regions close to shadows compared to the pre-perihelion images, the average spectral slope has not changed significantly even if it has a higher dispersion. We find a slope of $16.8 \pm 2.1$ [per cent $(100 \mathrm{~nm})^{-1}$ ] and $16.6 \pm 2.6$ [per cent $(100 \mathrm{~nm})^{-1}$ ] for the $7 \mathrm{~h} 31$ and $7 \mathrm{~h} 35$ images, respectively. These results are in agreement with the ones found by Fornasier et al. (2016), who reported a progressive blueing of the 67P nucleus' colours approaching the perihelion compared to the inbound observations at large heliocentric distance, followed by a reddening at post-perihelion distances $>2$ au. They interpreted this continuous colour changes of the nucleus along its orbit as due to the progressive thinning of the dust mantle when the comet approached perihelion and became more active, partially exposing the underlying ice-enriched layers. In the post-perihelion phase, the cometary activity did not sustain removal of dust, and the thicker dust mantle covered and masked the spectrally bluer ice-enriched layers, giving a nucleus spectrophotometry with spectral slope as red as the one observed in the pre-perihelion phase at high heliocentric distance. A similar trend was recently observed also in the Southern hemisphere region Anhur, with a steep increase of the spectral slope in pre- and post-perihelion phase at large heliocentric distances compared to the observations close to the perihelion passage (Fornasier et al. 2017).

\section{CONCLUSIONS}

This work focused on the circular niches located in the Seth region on the body of 67P. By means of OSIRIS NAC and WAC images, we have performed a detailed analysis of the area using different resolutions. During one of the latest Rosetta elliptical orbits, a set of images were acquired giving a unique view of these interesting features at high resolution. Specifically, thanks to the WAC image taken on 2016 August 24 with a scale of $0.31 \mathrm{~m} \mathrm{px}^{-1}$, we made a geomorphological map of the area identifying different terrain units. These niches are characterized by the presence of both gravitational accumulation deposits and talus deposits that can be separated on the basis of their texture. Such deposits cover the terrain outlined by the adjacent outcropping walls, which are defined as another geomorphological unit.

Between the different features that can be identified in the image, the boulders field deposits mainly characterize the interior of the circular niches. Thanks to the scale of the images we were able to identify boulders with different shapes with a diameter larger than $1 \mathrm{~m}$. After the identification of these features, we statistically analysed their distribution deriving a cumulative SFD with similar power-law indices for niches $\mathrm{A}$ and $\mathrm{B}$. The fitting regression lines returned two different power-law indices on the basis of the boulders dimension within the same deposit. Indeed, niche A is described by a power-law index equal to -2.3 for boulders diameter ranging from 1.8 and $5 \mathrm{~m}$, and a power-law index of -5.0 for boulders larger than $5 \mathrm{~m}$. Niche B is described by a similar behaviour consisting of a power-law index of -2.7 for boulders diameter ranging from 1.8 and $5 \mathrm{~m}$, and a power-law index of -4.7 for boulders larger than $5 \mathrm{~m}$. When comparing these results with the Pajola et al. (2015) work, we obtain that the values for smaller boulders (smaller than $5 \mathrm{~m}$ ) fall within the SFD case (ii) and (iii), while larger boulders (larger than $5 \mathrm{~m}$ ) can be related to the SFD case (i). This suggests that smaller boulders have formed during gravitational collapses, but have been progressively degraded and depleted lowering the power-law index and, in addition, the degradation of smaller boulders might have concurred to determine this SFD trend. The behaviour found for larger boulders, that are located in proximity of highly fractured 

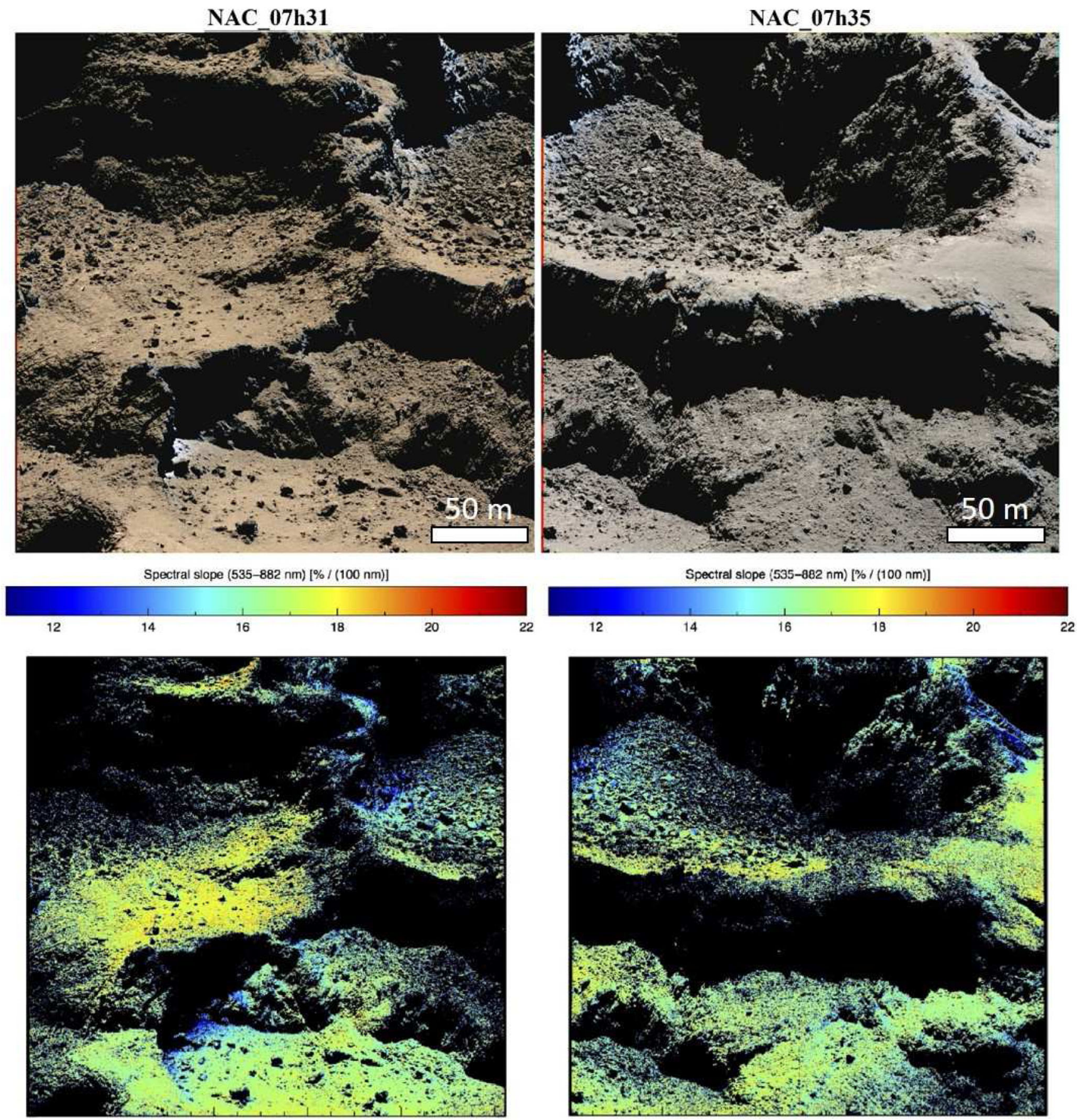

Figure 10. Upper panel: RGB images, in false colours, of the investigated region for images taken on 2016 July 23 with filters centred on 480,649 and $882 \mathrm{~nm}$. Rosetta was at a distance of $7.9 \mathrm{~km}$ resulting in a scale of $0.15 \mathrm{~m} \mathrm{px}^{-1}$. On the left is the NAC image acquired at UT time $07 \mathrm{~h} 31$ showing parts of niches A, B and C, while on the left is the NAC image acquired at UT time 07h35 displaying the niches A and C. Some frosts/water ice enriched regions are seen close to the shadows or in shadowed regions (regions with relatively bluer colours). Lower panel: spectral slope of the corresponding images at a phase angle of $89^{\circ}$. The slope is computed in the $535-882 \mathrm{~nm}$ range, after the normalization at $535 \mathrm{~nm}$ and it is in per cent $(100 \mathrm{~nm})^{-1}$.

walls, can be the expression of an enhanced fragmentation, which might be produced during the depression formation that caused the release of pressurized volatiles. To detect if there have been any geomorphological changes, we used a NAC image taken before perihelion in 2015 March to compare the distribution of boulders within the niches. In particular, we found similar pre/post results for niche A implying no specific changes in the area. In addition, from the erosion model characterizing the niches it must be noted that the area is not affected by strong erosion over one comet period, hence, we did not expect large variations on the different geological features. After identifying multiple terrains with different kinds of textures on Seth's niches, we performed the spectral analysis of this region in order to understand if there are any spectrophotometric differences between the various geomorphological units. We performed the spectrophotometric analysis for pre- and post-perihelion images to detect possible colour changes in the area. For this 

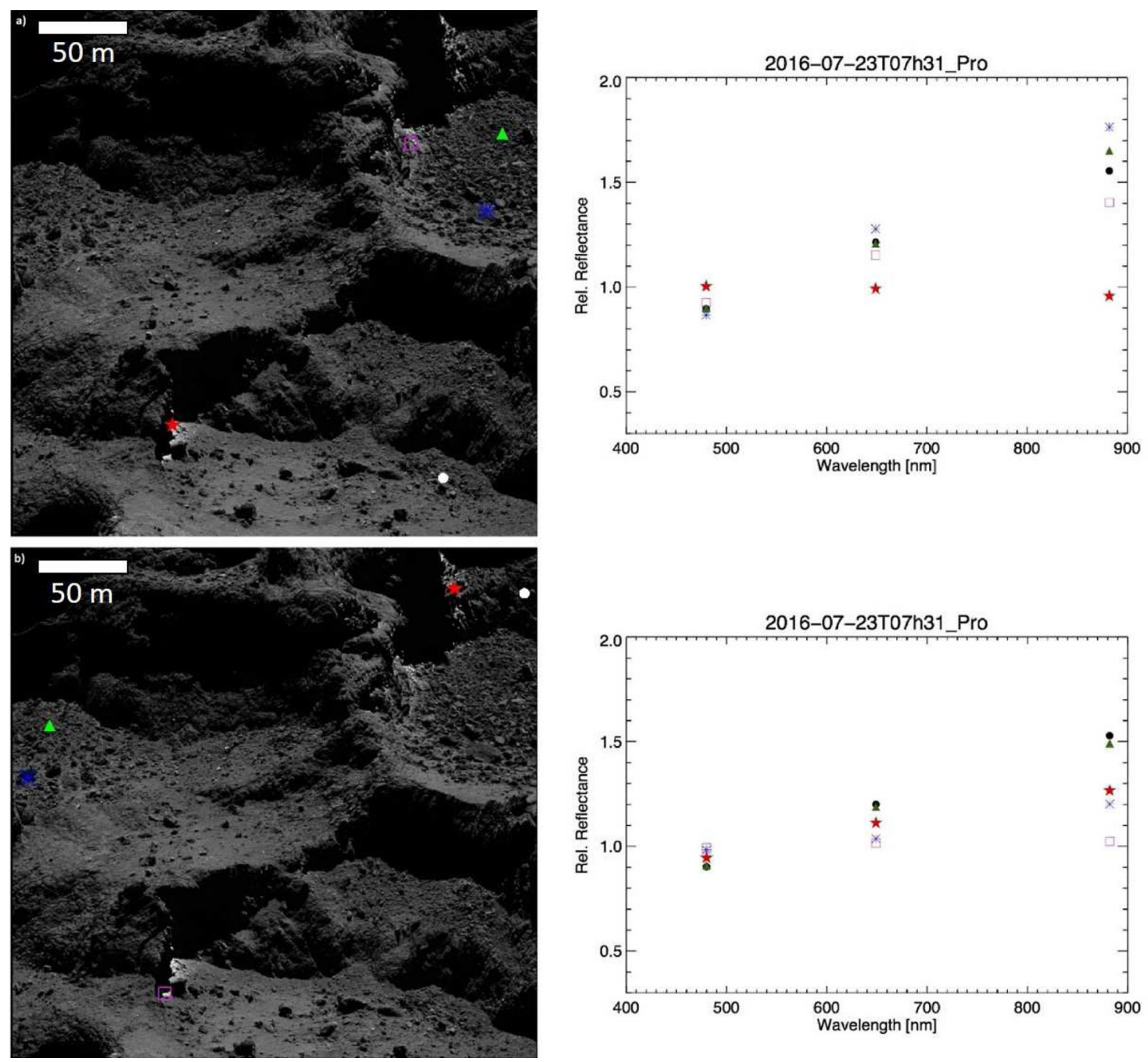

Figure 11. On the left are shown 10 ROIs of the NAC $07 \mathrm{~h} 31$ UT where the spectrophotometry has been computed. On the right, the relative reflectance for the corresponding selected regions is displayed. As the green filter is not available, the normalization is done by linear fitting and interpolation using the 649 and $480 \mathrm{~nm}$ filter images. The black filled circle on the right is represented by a white circle on the image on the left.

purpose, we evaluated the spectral slope for data sets acquired in 2014 November and 2016 July, whose images have different resolutions ( 0.84 and $0.15 \mathrm{~m}$ for the pre- and post-perihelion, respectively) but similar geometry. We also identified and analysed different ROIs that sample many areas of the niches (16 ROIs for pre-perihelion data set and 20 ROIs for post-perihelion data sets). Comparing the results, we found that the average spectral slope has not changed significantly after the Rosetta perihelion passage, but we observed bluer spots in images taken after perihelion indicating the presence of exposed water ice mixed to the refractory materials, in agreement with what was previously observed in different regions of the nucleus (Fornasier et al. 2016; Oklay et al. 2016a; Oklay et al. 2017; Pajola 2017).

The overall analysis implies minor colour changes in images with a spatial resolution of $15 \mathrm{~cm} \mathrm{px}^{-1}$, while geomorphological variations have not been observed, maybe because the expected erosion was below the detection limit, i.e. the spatial resolution of images used for geomorphological analysis. No specific changes in boulders deposits suggest that the gravitational event that gave birth to these deposits is not related to the recent detected activity of 67P. This means that the landslide that originated the boulders deposits occurred in the past, maybe implying that these areas can be older than the nearby ones. This is also in agreement with El-Maarry (2017)'s work in which the analysis of detected surface changes imply a more active comet in the past.

\section{ACKNOWLEDGEMENTS}

Optical, Spectroscopic and Infrared Remote Imaging System (OSIRIS) was built by a consortium of the Max-Planck-Institut für Sonnensystemforschung, in Göttingen, Germany, CISASUniversity of Padova, Italy, the Laboratoire d'Astrophysique de Marseille, France, the Instituto de Astrofisica de Andalucia, CSIC, Granada, Spain, the Research and Scientific Support Department of the European Space Agency, Noordwijk, the Netherlands, the Instituto Nacional de Tecnica Aeroespacial, Madrid, Spain, the Universidad Politechnica de Madrid, Spain, the Department of Physics and Astronomy of Uppsala University, Sweden and the Institut für Datentechnik und Kommunikationsnetze der Technischen Universität Braunschweig, Germany. The support of the national funding agencies of Germany (DLR), Italy (ASI), France (CNES), Spain (MEC), Sweden (SNSB) and the ESA Technical Directorate is 

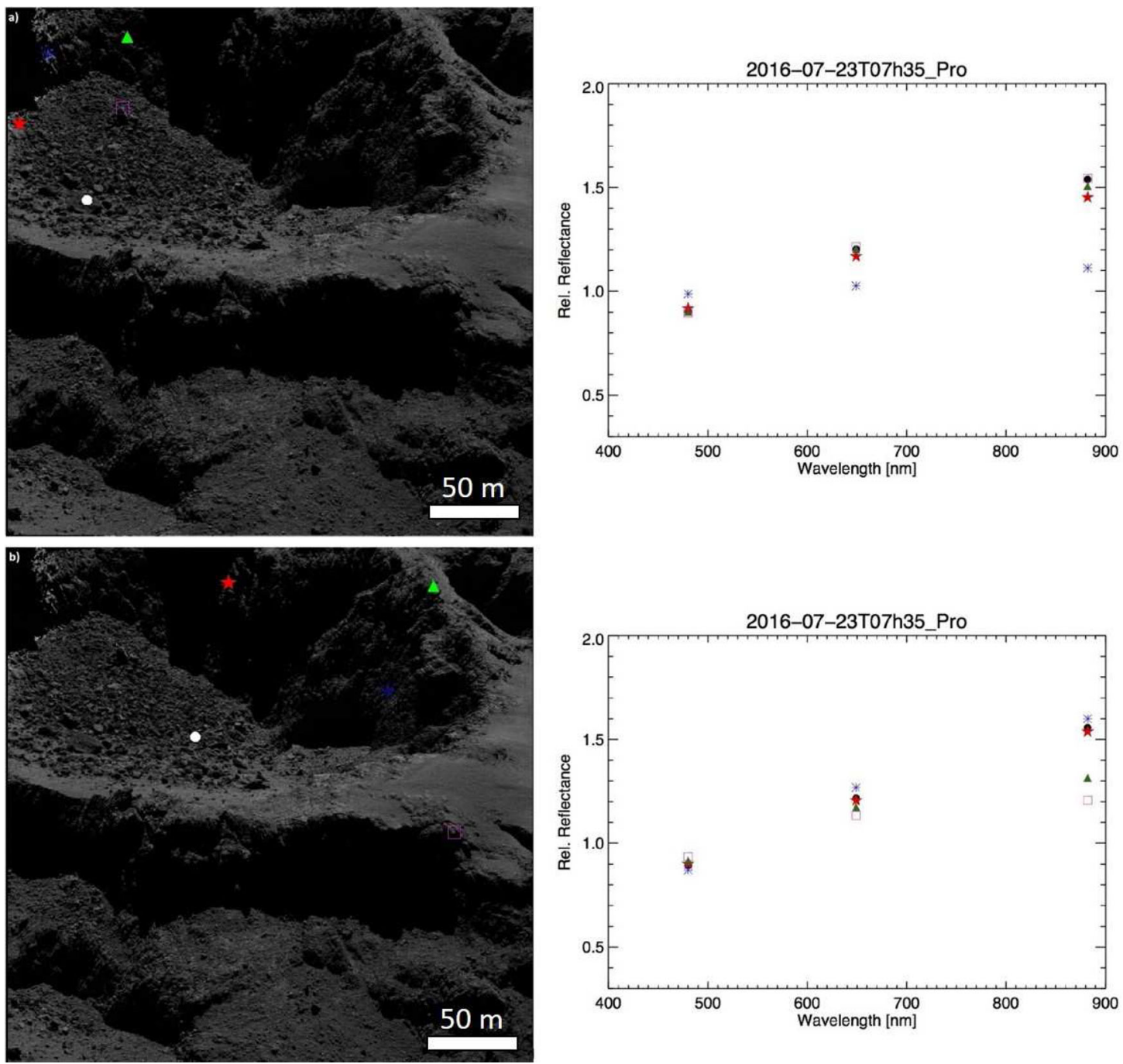

Figure 12. On the left are shown 10 selected ROIs of the NAC $07 \mathrm{~h} 35$ UT where the spectrophotometry has been computed. On the right, the relative reflectance for the corresponding selected regions is displayed. As the green filter is not available, the normalization is done by linear fitting and interpolation using the 649 and $480 \mathrm{~nm}$ filter images. The black filled circle on the right is represented by a white circle on the image on the left.

gratefully acknowledged. We thank the ESA teams at ESAC, ESOC and ESTEC for their work in support of the Rosetta mission. We made use of the ARCGIS 10.2 software together with IDL and MATLAB software to perform the presented analysis.

\section{REFERENCES}

Barucci M. A. et al., 2016, A\&A, 595, A102

Deshapriya J. D. P. et al., 2016, MNRAS, 462, S274

El-Maarry M. R. et al., 2017, Science, 355, 1392

El-Maarry M. R. et al., 2015a, Geophys. Res. Lett., 42, 5170

El-Maarry M. R. et al., 2015b, A\&A, 583, A26

Farneback G., 2001, Proc. Eighth IEEE Int. Conf. on Comput. Vis. ICCV 2001, Very High Accuracy Velocity Estimation Using Orientation Tensors, Parametric Motion, and Simultaneous Segmentation of the Motion Field. IEEE Computer Society Press, Vancouver, BC, p. 171

Feller C. et al., 2016, MNRAS, 462, S287

Filacchione G. et al., 2016, Nature, 529, 368

Fornasier S. et al., 2015, A\&A, 583, A30

Fornasier S. et al., 2016, Science, 354, 1566

Fornasier S. et al., 2017, MNRAS, 469, S93

Giacomini L. et al., 2016, MNRAS, 462, S352
Groussin O. et al., 2015, A\&A, 583, A36

Hapke B., 1993, Theory of Reflectance and Emittance Spectroscopy. Cambridge Univ. Press, Cambridge

Jorda L. et al., 2016, Icarus, 277, 257

Keller H. U. et al., 2007, Space Sci. Rev., 128, 433

Keller H. U. et al., 2015, A\&A, 583, A34

La Forgia F. et al., 2015, A\&A, 583, A41

Lucchetti A. et al., 2016, A\&A, 585, L1

Massironi M. et al., 2015, Nature, 526, 402

Mazrouei S., Daly M. G., Barnouin O. S., Ernst C. M., DeSouza I., 2014, Icarus, 229, 181

Michikami T. et al., 2008, Earth Planets Space, 60, 13

Nyquist H., 1928, 47, 617

Oklay N. et al., 2016a, MNRAS, 462, S394

Oklay N. et al., 2016b, A\&A, 586, A80

Oklay N. et al., 2017, MNRAS, this issue

Pajola M. et al., 2017, Nature Astron., 1, 0092

Pajola M. et al., 2015, A\&A, 583, A37

Pajola M. et al., 2016a, MNRAS, 462, S242

Pajola M. et al., 2016b, A\&A, 585, A85

Pajola M. et al., 2016c, A\&A, 592, L2

Pajola M. et al., 2016d, A\&A, 592, A69

Pommerol A. et al., 2015, A\&A, 583, A25 
Preusker F. et al., 2015, A\&A, 583, A33

Rossi A., Fulchignoni M., 1999, Planet. Space Sci., 47, 873

Sierks H. et al., 2015, Science, 347, aaa1044

Thomas N. et al., 2015, Science, 347, aaa0440

Tubiana C. et al., 2015, A\&A, 583, A46

van der Walt S. et al., 2014, preprint (arXiv:1407.6245)

Vincent J.-B. et al., 2015, Nature, 523, 63

Werner R. A., Scheeres D. J., 1997, Celest. Mech. Dyn. Astron., 65, 313

${ }^{1}$ INAF - OAPD Astronomical Observatory of Padova, Vic. Osservatorio 5, I-35122 Padova, Italy

${ }^{2}$ NASA Ames Research Center, Moffett Field, CA 94035, USA

${ }^{3}$ LESIA-Observatoire de Paris, PSL Research University, CNRS, Univ. Paris-Diderot, Sorbonne Paris Cite, UMPC Univ. Paris 06, Sorbonne universites, 5 Place J. Janssen, F-92195 Meudon Principal Cedex, France

${ }^{4}$ Deutsches Zentrum für Luft- und Raumfahrt (DLR), Institut für Planetenforschung, Rutherfordstrasse 2, D-12489 Berlin, Germany

${ }^{5}$ Center of Studies and Activities for Space, CISAS, 'G. Colombo', University of Padova, Via Venezia 15, I-35131 Padova, Italy

${ }^{6}$ Laboratoire d'Astrophysique de Marseille, UMR 7326 CNRS \& AixMarseille Université, 38 rue Frédéric Joliot-Curie, F-13388 Marseille cedex 13, France

${ }^{7}$ Geosciences Department, University of Padova, Via G. Gradenigo 6, I-35131 Padova, Italy

${ }^{8}$ Department of Information Engineering, University of Padova, Via Gradenigo 6/B, I-35131 Padova, Italy

${ }^{9}$ CNR-IFN UOS Padova LUXOR, Via Trasea 7, I-35131 Padova, Italy

${ }^{10}$ Max-Planck-Institut für Sonnensystemforschung, Justus-von-Liebig-Weg, 3 D-37077 Göttingen, Germany

${ }^{11}$ Department of Physics and Astronomy 'G. Galilei', University of Padova, Vic. Osservatorio 3, I-35122 Padova, Italy

${ }^{12}$ Centro de Astrobiologa, CSIC-INTA, E-28850 Torrejon de Ardoz, Madrid, Spain
${ }^{13}$ International Space Science Institute, Hallerstrasse 6, CH-3012 Bern, Switzerland

${ }^{14}$ Scientific Support Office, European Space Research and Technology Centre/ESA, Keplerlaan 1, Postbus 299, NL-2201 AZ Noordwijk ZH, the Netherlands

${ }^{15}$ Department of Physics and Astronomy, Uppsala University, E-75120 Uppsala, Sweden

${ }^{16}$ PAS Space Research Center, Bartycka 18A, PL-00716 Warszawa, Poland

${ }^{17}$ Institute for Geophysics and Extraterrestrial Physics, TU Braunschweig, D-38106 Braunschweig, Germany

${ }^{18}$ Department for Astronomy, University of Maryland, College Park, MD 20742-2421, USA

${ }^{19}$ LATMOS, CNRS/UVSQ/IPSL, 11 Boulevard d'Alembert, F-78280 Guyancourt, France

${ }^{20}$ NASA Jet Propulsion Laboratory, 4800 Oak Grove Drive, Pasadena, CA 91109, USA

${ }^{21}$ Department of Mechanical Engineering, University of Padova, Via Venezia 1, I-35131 Padova, Italy

${ }^{22}$ UNITN, University of Trento, Via Mesiano, 77, I-38100 Trento, Italy

${ }^{23}$ INAF Osservatorio Astronomico di Trieste, Via Tiepolo 11, I-34143 Trieste, Italy

${ }^{24}$ Instituto de Astrofisica de Andalucia CSIC, Glorieta de la Astronomia, E-18008 Granada, Spain

${ }^{25}$ Institute for Space Science, National Central University, 32054 Chung-Li, Taiwan

${ }^{26}$ Operations Department European Space Astronomy Centre/ESA, PO Box 78, E-28691 Villanueva de la Canada, Madrid, Spain

${ }^{27}$ Physikalisches Institut der Universität Bern, Sidlerstr. 5, CH-3012 Bern, Switzerland

This paper has been typeset from a $\mathrm{T}_{\mathrm{E}} \mathrm{X} / \mathrm{L} \mathrm{T} \mathrm{T}_{\mathrm{E}} \mathrm{X}$ file prepared by the author. 